Received: 15 January 2018

Accepted: 19 March 2018

Published online: 03 April 2018
CIENTIFIC REP

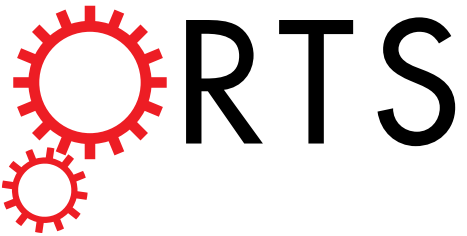

\section{OPEN Water-Soluble Metalated Covalent Organic Nanobelts with Improved Bioavailability for Protein Transportation}

\author{
Weifu Kong ${ }^{1}$, Jiaxun Wan ${ }^{1}$, Supawadee Namuangruk ${ }^{2}$, Jia Guo ${ }^{1}{ }^{1} \&$ Changchun Wang ${ }^{1}$
}

An available pathway to prepare the ionized covalent organic nanosheets (iCONs) has been proposed by a metal-assisted aqueous-phase exfoliation route from covalent organic frameworks. The soluble and belt-shaped iCONs could immobilize a large quantity of proteins $(2.73 \mathrm{mg} / \mathrm{mg}, \mathrm{BSA} / \mathrm{iCONs})$ and hence serve as transporters to enhance the protein uptake by cancer cells. Meanwhile, their energy-dependent endocytosis pathway via clathrin-coated pits has been proved as well.

Recent decades have witnessed enormous progress on covalent organic frameworks (COFs), which owe many of fantastic properties to their crystalline, porous and layered structures with intrinsic two-dimensional atomic frameworks ${ }^{1}$. Minimization of bulky COFs into the nanometer scale hold great promises for medical and diagnostic applications. Several groups have demonstrated the potent applicability of COFs in drug loading and sustained release in $v$ tro $^{2-5}$. Modification of exfoliated COFs with specific receptors also could implement the target delivery of loaded drugs ${ }^{6}$. In addition to shuttle cargoes, nanoscale COFs have been actively pursued as a brand-new platform for phototherapy ${ }^{7}$, enzyme immobilization ${ }^{8}$, and biomolecular detection ${ }^{9}$. However, the early reports have rarely investigated the fundamental interactions of COFs with living systems for improving the transportation ability of these materials. Meanwhile, such a COF-mediated cellular uptake mechanism and pathway remain, for a large part, unknown yet.

To access the cellular internalization, preparation of dispersible and discrete nanoscale COFs is a prerequisite for in vitro test. There are two main strategies reported thus so far for design of bioavailable COFs. One is the synthetic control over size and morphology of COF crystallite assembles that could have a diversity of forms such as microspheres, nanofibers, nanoplates and so on ${ }^{10}$. The other is the delamination of 2D COFs into single or few-layered covalent organic nanosheets (CONs) by destructing the relatively weak $\pi-\pi$ interactions within neighboring layers. Comparably, the exfoliated ultrathin COFs are assumed to benefit cellular internalization because $2 \mathrm{D}$ organic layers could provide abundant surface and edge functionality as well as low-dimensional strength for ease of migration across cell membranes. Direct exfoliation of aromatic COFs using mechanical forces (e.g. grinding ${ }^{11}$ and ball milling ${ }^{12}$ ) in solid state or liquid-based ultra-sonication ${ }^{13-15}$ is the commonly used strategy, while such treated CONs are poorly dispersed in aqueous solution if without functional decoration. To render COFs hydrophilic, ionization of main backbones has been proposed, and the charged frameworks favor either the self-exfoliation ${ }^{16}$ or interfacial evolution ${ }^{17}$ into layered CONs. However, with this method there is an emerging need to pay more attention on challenges regarding synthesis of ionized subunits and COFs/CONs, which hence limits the development of COFs towards bio-application. Herein, we address a facile method to delamination and ionization of pristine keto-enamine-linked COFs and further immobilize fluorescence-labeled proteins for investigation of its cellular uptake mechanism.

\section{Results and Discussion}

As shown in Fig. 1a, the model reaction of 1,3,5-triformylphloroglucinol (Tp) with aniline derivatives results in $\mathrm{C}_{3 \mathrm{~h}}$ symmetric compounds that are normally considered a hybrid of keto-enamine form and enol-imine form ${ }^{18}$. The former tautomer has a main contribution to conjugate with metals ${ }^{19}$. Therefore, without incorporation of

${ }^{1}$ State Key Laboratory of Molecular Engineering of Polymers, Department of Macromolecular Science, and Laboratory of Advanced Materials, Fudan University, Shanghai, 200433, P.R. China. ${ }^{2}$ National Nanotechnology Center (NANOTEC), National Science and Technology Development Agency, Pathumthani, 12120, Thailand. Correspondence and requests for materials should be addressed to J.G. (email: guojia@fudan.edu.cn) 
(a)

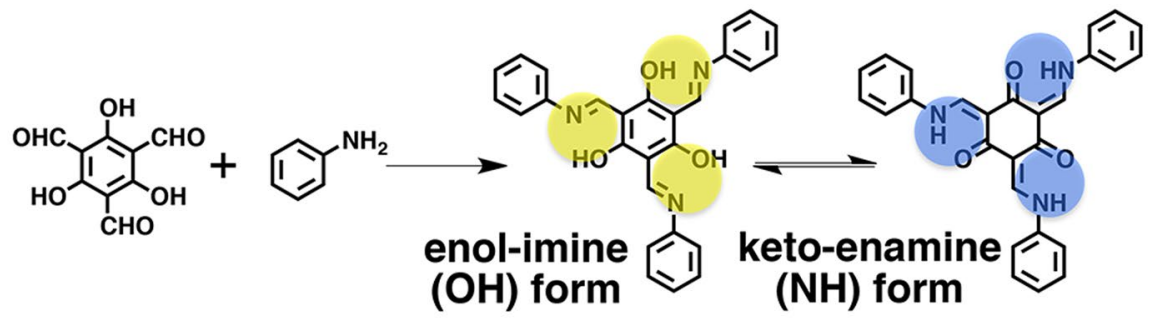

(b)<smiles>Nc1ccc(-c2ccc(N)cc2)cc1</smiles>

or

$\mathrm{NH}_{2}-\mathrm{NH}_{2}$

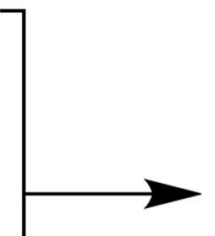

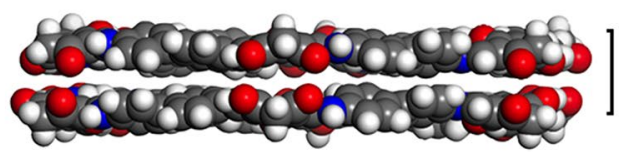

\section{Interaction energy: $-\mathbf{3 6 2} \mathrm{kJ} / \mathrm{mol}$}

\section{$\mathrm{Fe}^{3+}$-triggered delamination in water}

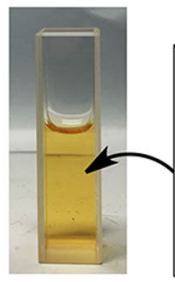

\section{Water-soluble CONs}

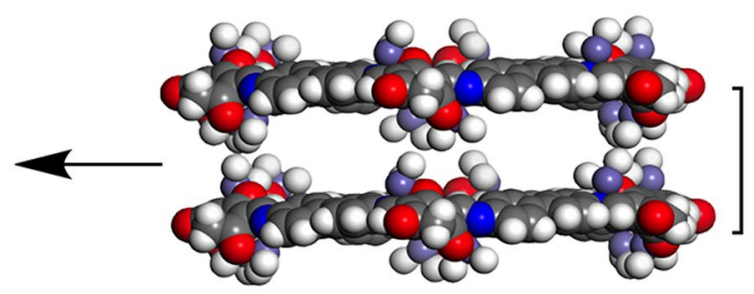

Interaction energy: $-\mathbf{1 9} \mathrm{kJ} / \mathrm{mol}$

Figure 1. Schematic representation of metal-assisted exfoliation of keto-enamine-linked COFs in aqueous solution. (a) Schiff-base reaction of Tp and aniline to produce the tautomers of tris $(N$-salicylideneaniline), wherein the chelation sites are shown in circles. (b) Calculation of interlayer distances and interaction energies before and after the Fe(III)-assisted aqueous-phase exfoliation of COF into the corresponding soluble CON. The inset is a photograph of the dispersion of CONs in aqueous solution.

ligand subunits (e.g. phthalocyanine, porphyrin, dehydrobenzoannulene, and salen) ${ }^{20-24}$, a purposeful design of the COF linkers could host metals on the edges of organic networks. Then we commenced on the exfoliation of keto-enamine-linked COFs containing $\mathrm{Fe}(\mathrm{III})$-coordinated tris $(\mathrm{N}$-salicylideneaniline) complexes. Prior to the experimental trials, theoretical calculations have been performed by the force field method to investigate the interaction of adjacent layers upon formation of Schiff-base complexes on COF backbones. One can see that the $\mathrm{Fe}$ atoms with their counterpart ions reside out of plane to make the single atomic layer thicker and increase the interlayer distance (Fig. 1b). In comparison of the pristine COFs, the interaction energy between layers is largely reduced to $-19 \mathrm{~kJ} / \mathrm{mol}$ along with increase of the interlayer distance of up to $9.85 \AA$. The weak electrostatic force is assumed to maintain the superimposition of layers on each other, while such the metalated COFs should be easily intercalated by solvents to result in the independent CONs. The emerging Schiff-base complexes on backbones would largely improve hydrophilicity and hence bioavailability of such materials, which thus are accessible to functional proteins or enzymes transported into cytoplasm by using the soluble CONs as vehicles. Compared with the other known routes, metal chelation of keto-enamine-linked COFs no longer requires either the purposeful design of ionized starting materials or chemically challenging synthesis of structurally complicated ionic COFs.

Polymerization of Tp with benzidine (BD) was conducted in a mixture of $o$-dichlorobenzene and $n$-butyl alcohol $(\mathrm{v} / \mathrm{v}, 9 / 1)$ at $120^{\circ} \mathrm{C}$ for $72 \mathrm{~h}$ under the typical solvothermal conditions. Analogue to the early reported, the obtained products held the hexagonal lattices, high surface areas, and typical microporosity as well as insolubility. Then the $\mathrm{COF}(\mathrm{TpBD})$ powders were dispersed in water with ultrasonic assistance, while the suspension was composed of large grains even with prolonged treatment. On dissolution of $\mathrm{FeCl}_{3}$ salts, the suspended grains were invisible after 1-h ultra-sonication (inset in Fig. 1b), and the Tyndall effect was observed in the resulting aqueous solution, indicating that the COF aggregates were striped into discrete and dispersible nanomaterials with sufficiently small sizes out of the visible range. With removal of the free Fe(III) ions by dialysis, the homogeneity of the aqueous solution was confirmed by dynamic light scattering measurement, giving a predominated diameter of roughly $50 \mathrm{~nm}$ as well as narrow size distribution of PDI $<0.3$ (Figure S1, Supplementary Information). 

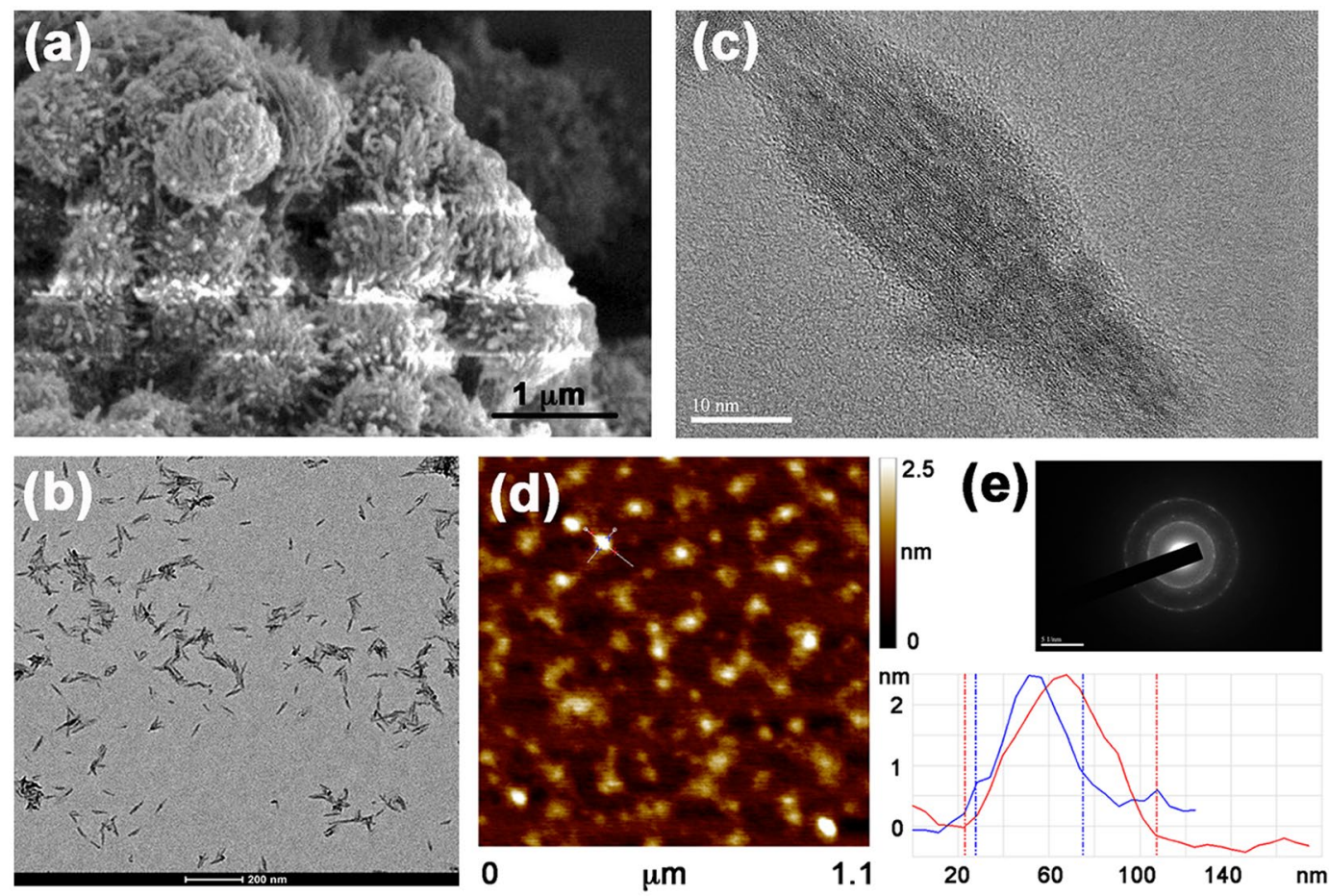

Figure 2. Morphology of belt-shaped CONs. (a-c) TEM image of bulk COF(TpBD) (a) and exfoliated $\mathrm{CON}(\mathrm{TpBD})(\mathbf{b})$ with its magnified view (c). (d) AFM image and the height profiles of exfoliated CON(TpBD). (e) SAED pattern of exfoliated CON(TpBD).

By following the same treatment pathway, $\mathrm{COF}(\mathrm{TpHA})$ synthesized by Tp with hydrazine (HA), could dissolve in water as well (Figure S2, Supplementary Information). We reason that the complexation of Fe(III) ions with tri( $N$-salicylideneaniline) units of COFs occur in the 1D pore channels, and in turn, the metalated COF skeletons are ionized and hydrated leading to the disassembly of aggregates and subsequent delamination of layered structures in water. Even without mechanical forces, the electrostatic repulsion also could offset the $\pi-\pi$ interaction of interlayers, forming dispersible nanomaterials just by letting it stand overnight. In contrast, if the imine-linked COFs evolved from the polymerization of benzene-1,3,5-tricarbaldehyde and BD, the weak coordination of $-\mathrm{C}=\mathrm{N}$ - with $\mathrm{Fe}(\mathrm{III})$ ions was insufficient to allow for the metal insertion and ionization of COF skeletons for aqueous-phase exfoliation. FT IR measurement confirmed that the characteristic bands of COF(TpBD)s were maintained in the structure of exfoliated products, except of the hydrated groups around $3400 \mathrm{~cm}^{-1}$ (Figure S3, Supplementary Information). Also, it was found that the yield of CONs was correlated with the added amount of $\mathrm{Fe}$ (III) ions, and could reach $>90 \%$ yields after the two runs of exfoliation procedure (Table S1, Supplementary Information).

SEM image of the as-synthesized COF(TpBD) in Fig. 2a reveals its clustering morphology formed with numerous nanofibers. After metal-assisted aqueous-phase exfoliation, discrete and uniform rod-like morphology is shown in TEM image, having an average length of around micron-meters (Fig. 2b). The magnification of the view on a single nanorod evidences the long-distance alignment at the molecular level (Fig. 2c and Figure S4, Supplementary Information), which is verified again by the polycrystalline diffraction rings displayed in the SAED pattern (Fig. 2e). However, the aqueous-phase XRD pattern of CON(TpBD) discloses its amorphous nature, as the main peak at $3.8^{\circ}$ corresponding to the (100) lattice plane disappears (Figure S5, Supplementary Information). The same observation has been reported as the hydrazone-linked COFs were exfoliated ${ }^{25}$, implying that the delaminated $\mathrm{CON}(\mathrm{TpBD})$ retained partially ordering. To characterize the sizes of $2 \mathrm{D}$ geometry, AFM was performed to identify a belt-like morphology that is of a lateral length of $c a .80 \mathrm{~nm}$ and a thickness of $c a .2 .5 \mathrm{~nm}$ (Fig. 2d), indicative of less than 10-layer sheets exfoliated out of the bulky COF grains. The method used here is appropriate for not only COF(TpBD), but also for the other COFs containing tri( $N$-salicylideneaniline) tautomers in the nodes of networks (Figure S6, Supplementary Information). The metal distribution was measured by the atomic mapping on the representative sample, wherein the abundant Fe atoms reside throughout the nanobelt and are overlapped with the other regions from $\mathrm{N}$ and $\mathrm{O}$ atoms (Figure S7, Supplementary Information).

In addition, the nitrogen sorption isotherms (Fig. 3a) were examined to estimate the surface area by using the Brunauer-Emmett-Teller (BET) model. Compared with the pristine COF(TpBD) $\left(1850 \mathrm{~m}^{2} / \mathrm{g}\right)$, the BET surface area of CON(TpBD) is largely reduced to be $117 \mathrm{~m}^{2} / \mathrm{g}$ (the detailed BET and Rouquerol plots are shown in Figures S8 and S9 in the Supplementary Information). The opening pores instead remain on the CON(TpBD), giving a broad pore-size distribution in the range of 2 to $10 \mathrm{~nm}$ (Figure S10, Supplementary Information).

To clarify the metalation mechanism, X-ray photoelectron spectroscopy provided insight into the binding energies of different atoms. As shown in Fig. 3b, a double broad peak from the Fe2p signal is deconvoluted into 

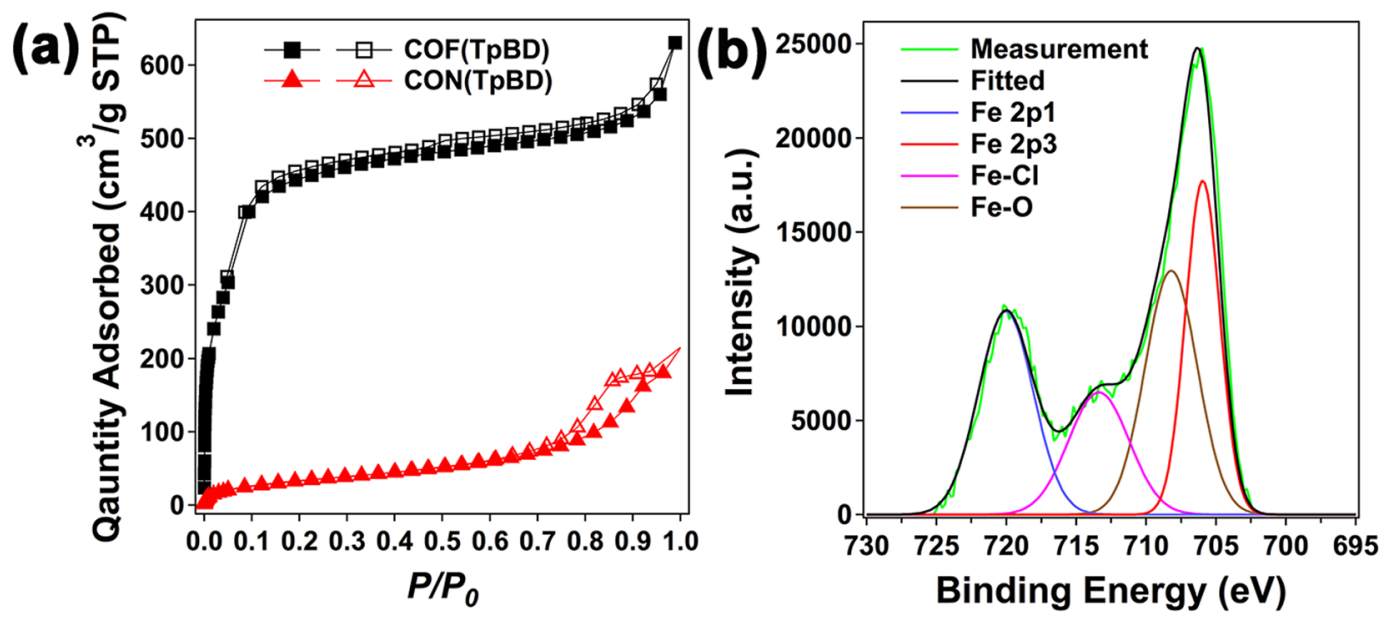

Figure 3. Measurements of porosity and atomic binding energy. (a) $\mathrm{N}_{2}$ sorption isotherms of COF(TpBD) and CON(TpBD). (b) XPS spectrum of Fe2p signal from CON(TpBD).

four individual peaks at $720 \mathrm{eV}, 706 \mathrm{eV}, 713 \mathrm{eV}$, and $708 \mathrm{eV}$, which are assignable to $\mathrm{Fe} 2 \mathrm{p} 1, \mathrm{Fe} 2 \mathrm{p} 3, \mathrm{Fe}-\mathrm{Cl}$, and Fe-O units, respectively. This reveals that the dominant keto-enamine structures ${ }^{26}$ could conjugate with $\mathrm{Fe}$ (III) ions in HN...O pockets. On the other hand, it was detected that $26 \mathrm{wt} . \%$ of Fe atoms is immobilized into the CONs. The calculated molar ratio of Fe and tri(N-salicylideneaniline) units is close to 3:1, indicating the formation of trinuclear Fe(III) complexes. For extension of the available metal species, $\mathrm{Fe}$ (II), $\mathrm{Zn}$ (II) and $\mathrm{Cu}$ (II) ions were testified to exfoliate the keto-enamine-linked COFs, whereas they didn't work with the identical operation. We reason that some kinds of the metal complexes formed on COF skeletons cannot attenuate the $\pi-\pi$ interaction between the neighboring layers.

Prior to the study of intracellular uptake pathway for the protein-loaded CONs, we estimated the cytotoxicity and intracellular distribution of CON(TpBD) in Hep G2 cancer cells. As observed in Fig. 4b, nearly no Hep G2 cells are dead at the maximum concentration of $2000 \mu \mathrm{g} / \mathrm{mL}$, and over $100 \%$ viability could be maintained after $48 \mathrm{~h}$. To access the intracellular distribution of transporters, the residual amino groups on the periphery of CONs were employed to react with fluorescein isothiocyanate (FITC) to label the CON materials. The FITC-modified CON shows a strong fluorescence emission around $513 \mathrm{~nm}$ with an excitation wavelength of $490 \mathrm{~nm}$ (Figure S11, Supplementary Information). After a 2-h incubation with Hep G2 cells, the CON probes accumulate within cells, and the produced green fluorescence is mainly distributed around the cell nucleus (Figure S12, Supplementary Information).

In view of metalated and porous interfaces of the soluble CONs, FITC-labeled BSA proteins could be readily adsorbed on it by the electrostatic interaction. The aqueous dispersion of FITC-BSA@CONs displays brilliant green fluorescence with UV lamp irradiation (Fig. 4a). The loaded BSA protein reaches as high as $2.73 \mathrm{mg}$ per milligram of CONs in PBS solution evaluated by the standard curve of FITC-BSA (Figure S13, Supplementary Information). To our knowledge, the protein-loading efficiency of CONs is much higher than those of the analogous $2 \mathrm{D}$ or porous materials such as oxidized graphene $(1.5-1.8 \mathrm{mg} / \mathrm{mg})^{27-29}$, and MOFs $(0.18 \mathrm{mg} / \mathrm{mg})^{30}$. In sharp contrast, the bulky COF powders have the relatively low loading capacity $(0.54 \mathrm{mg} / \mathrm{mg}, \mathrm{BSA} / \mathrm{COF})$, mainly because the BSA protein has so large dimension that nanopores of COFs $(1 \sim 3 \mathrm{~nm})$ are incapable of immobilizing them.

Next, we underwent a rigorous investigation of the cellular internalization mechanism and pathway for CON-based conjugates. In general, low-molecular-weight solutes transport through the plasma membrane via the energy-independent pathway. Relatively large particles are internalized by the energy-dependent pathway, also known as endocytosis that could be hindered when incubation are performed at $4{ }^{\circ} \mathrm{C}$ or in adenosine triphosphate (ATP)-free medium ${ }^{31}$. ATP production in cells can be disturbed with $\mathrm{NaN}_{3}$, thus blocking the endocytotic pathway. Along this line, Hep G2 cellular incubations with FITC-BSA@CON conjugates were carried out at $4{ }^{\circ} \mathrm{C}$ and with the cells pretreated with $\mathrm{NaN}_{3}$ for $1.5 \mathrm{~h}$, respectively, and the cells incubated at $37^{\circ} \mathrm{C}$ without any pre-treatment were used as a control group. Indeed, a low fluorescence level detected by flow cytometry measurement was obtained for the cells incubated at $4^{\circ} \mathrm{C}$ and in the presence of $\mathrm{NaN}_{3}$, respectively. This indicates endocytosis as the internalization mechanism for the uptake of BSA-CON at $37^{\circ} \mathrm{C}$. Then we studied the subcategories of the endocytosis pathway, which usually refer to phagocytosis, pinocytosis, and clathrin-dependent receptor-mediated and clathrin-independent endocytosis ${ }^{32}$. Of varieties, the most common pathway is receptor-mediated endocytosis, which occurs when the clathrin coat on the plasma membrane forms invaginations in the membrane to trap the extracellular species ${ }^{33}$. To access the role of clathrin in the uptake of CON conjugates, hypertonic treatment with sucrose was carried out to disrupt the formation of clathrin-coated vesicles on the cell membrane. It was observed that the fluorescence reduced when the cells were pretreated with sucrose, suggesting that the entry of BSA@CON conjugates into Hep G2 cells is mainly through a clathrin-dependent endocytosis pathway. However, the protein-CON conjugates was observed inside the cells even at $4{ }^{\circ} \mathrm{C}$ or in the presence of $\mathrm{NaN}_{3}$, which indicates that CON can partly enter into cells directly through an energy-independent pathway involving insertion and diffusion across the cell membrane. The enhanced internalization ability implies the superiority of low-dimensional CON as protein transporter. 


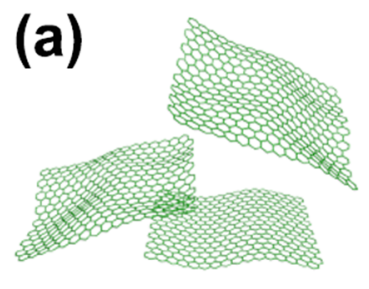

\section{Water-soluble CON}

\section{BSA protein with FITC tag \\ BSA Dimension: $14^{\star} 4^{\star} 4 \mathrm{~nm}$}
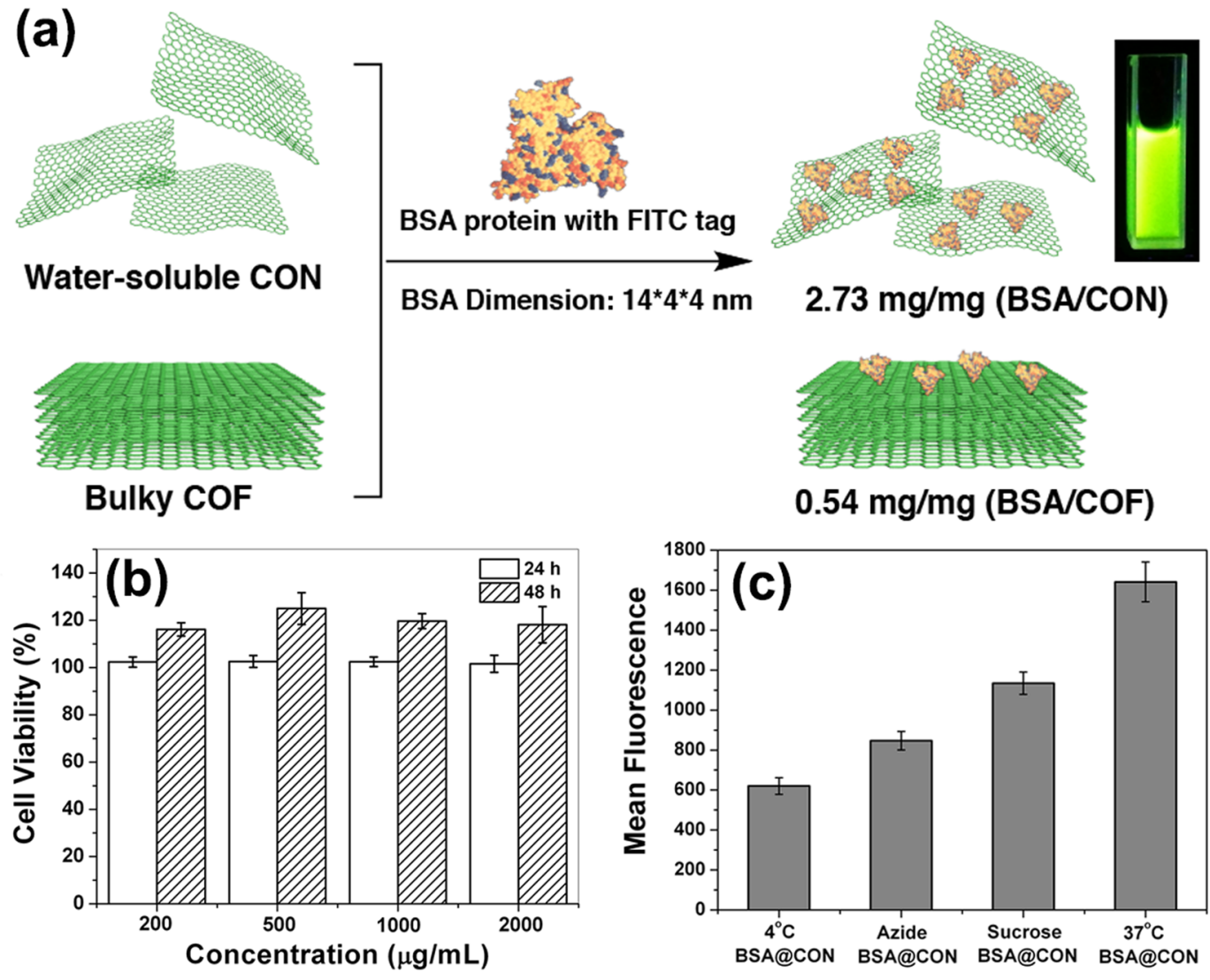

Figure 4. Loading and transportation of FITC-labeled BSA proteins with non-cytotoxic CONs. (a) Schematic representation of loading BSA-FITC proteins on CONs and COFs, and the fluorescent image shows the dispersion of FITC-BSA@CON in PBS solution under UV lamp. (b) Cytotoxicity test of CON(TpBD) incubated with Hep G2 cells for $24 \mathrm{~h}$ and $48 \mathrm{~h}$, respectively. (c) Flow cell cytometry data of Hep G2 cells after incubation in FITC-BSA@CON conjugates with pretreatment at $4{ }^{\circ} \mathrm{C}$ or with $\mathrm{NaN}_{3}$ or sucrose. The regular group was incubated at $37^{\circ} \mathrm{C}$ without any pretreatment.

\section{Conclusion}

In summary, metalated covalent organic nanosheets with few-layer molecular thickness is prepared by an aqueous-phase exfoliation method, which has been proved facile, efficient, and eco-friendly for disassembly of the pristine keto-enamine-linked COFs. The obtained CONs display uniform belt shape, water solubility and metal coordination as well as partial periodicity and porosity in structure. The bioavailability of CONs has been substantially proved by its non-cytotoxicity and enhanced loading capacity of proteins. We further have demonstrated that these ionized CONs are capable of the transportation of proteins into living cells and that the cellular-uptake mechanism is energy-dependent endocytosis, which occurs mainly through clathrin-coated pits. Establishment of the entry mechanism is of fundamental importance and will facilitate future developments of COF-related transporters for biological delivery applications.

\section{Materials and Methods}

Materials. 1,3,5-Trihydroxybenzene (THB) was purchased from Adamas Reagent Company. Trifluoroacetic acid (TFA), benzidine (BD), pyrrolidine, hydrazine hydrate, $o$-dichlorobenzene (DCB), fluorescein isothiocyanat (FITC) and $n$-butanol were purchased from Aladdin Industrial Corporation. Hexamethylenetetramine (HMT), acetic acid (HOAc), iron(III) chloride hexahydrate $\left(\mathrm{FeCl}_{3} \bullet 6 \mathrm{H}_{2} \mathrm{O}\right)$, and acetone were purchased from Shanghai Chemical Reagents Company. All reagents were purchased and used as received. Deionized water was used in all experiments.

Synthesis of 1,3,5-triformylphloroglucinol. According to a modified method presented by Foster et al. ${ }^{34}$, hexa-methylenetetramine $(15.10 \mathrm{~g}, 108 \mathrm{mmol})$ and phloroglucinol were added to $90 \mathrm{~mL}$ of trifluoroacetic acid under $\mathrm{N}_{2}$ atmosphere, and the solution was heated to $100^{\circ} \mathrm{C}$ and maintained for $2.5 \mathrm{~h}$. Then $150 \mathrm{~mL}$ of $3 \mathrm{M}$ $\mathrm{HCl}$ aqueous solution was mixed to allow for another 1 - $\mathrm{h}$ reaction at $100^{\circ} \mathrm{C}$. After being cooled to room temperature, the mixture was filtered through Celite, extracted with ca. $350 \mathrm{~mL}$ dichloromethane, and dried over $\mathrm{MgSO}_{4}$. After rotary evaporation, $4.19 \mathrm{~g}$ off-white powder was obtained in $19 \%$ yield. ${ }^{1} \mathrm{H} \mathrm{NMR}\left(400 \mathrm{MHz}, \mathrm{CDCl}_{3}\right): \delta$ (ppm) $14.12(-\mathrm{CHO}, \mathrm{s}, 1 \mathrm{H}), 10.18(-\mathrm{OH}, \mathrm{s})$. 
Synthesis of keto-enamine-linked COFs. According to the typical solvothermal protocol ${ }^{26,35}$, the keto-enamine-linked COFs were synthesized in a sealed vial, wherein 1,3,5-triformylphloroglucinol (10.5 mg, $0.05 \mathrm{mmol}$ ) and benzidine $(13.8 \mathrm{mg}, 0.075 \mathrm{mmol})$ were partially dissolved in a mixture of o-dichlorobenzene and $\mathrm{n}$-butyl alcohol (v/v, 9:1, $1 \mathrm{~mL})$ with addition of the aqueous solution of acetic acid $(6 \mathrm{M}, 0.1 \mathrm{~mL})$. The reaction mixture was subjected to a freeze-pump-thaw process for 3 cycles, and the sealed vial was kept in an oven at $120^{\circ} \mathrm{C}$ for $72 \mathrm{~h}$. After reaction, the solids were washed by acetone for three times in Buchner funnel. The target $\mathrm{COF}(\mathrm{TpBD})$ powders were obtained in $75 \%$ yield. Following the same steps, the $\mathrm{COF}(\mathrm{TpHA})$ solids were also obtained in $70 \%$ yield, using 1,3,5-triformylphloroglucinol $(10.5 \mathrm{mg}, 0.05 \mathrm{mmol})$ and hydrazine hydrate $(3.25 \mu \mathrm{L}$, $0.075 \mathrm{mmol}$ ).

Preparation and modification of CONs. The solid COF(TpBD) ( $2 \mathrm{mg}$ ) was added in the aqueous solution of $\mathrm{FeCl}_{3}$ salt $(3.8 \mathrm{mg}, 4 \mathrm{~mL})$. The $\mathrm{COF}$ grains were initially suspended in solution. After sonication for $1 \mathrm{~h}$, they could be gradually dissolved, and the solution turned transparent and yellow colour. The residual solids were then collected by centrifugation and subjected to the same treatment in a fresh $\mathrm{FeCl}_{3}$ aqueous solution $(4 \mathrm{~mL})$. The obtained CON solutions were combined to eliminate the free iron salts by dialysis, and freeze-dried to obtain the yellowish powder with $>90 \%$ yield. To modify the obtained CON(TpBD)s with fluorescent dyes, the isothiocyanate group of FITC molecules were reacted with the residual amino groups located on the periphery of nanosheets in ethanol at $70^{\circ} \mathrm{C}$ overnight. After reaction, the free FITC was removed by dialysis in ethanol, and the aqueous dispersion of FITC-modified $\mathrm{CON}(\mathrm{TpBD})$ was freeze-dried for further use.

Theoretical calculation. Compass (condensed-phase optimized molecular potentials for atomistic simulation studies) ${ }^{36}$ force-field included in the Forcite module of the Material Studio package was used to calculate the interlayer distances and interaction energies $\left(E_{\text {int }}\right)$ between the two adjacent layers of COF before and after insertion of $\mathrm{Fe}(\mathrm{III})$ ions and complexation. The systems were optimized in gas phase without any constraint. Convergence criteria of all the calculations were determined according to the ultrafine settings of the program. Partial charges on the atoms were conducted by the force field assigned charging methods that was included in the Compass force fields. The interaction energy of the two neighboring layers is expressed by

$$
E_{\text {int }}=E_{2 \mathrm{COF}}-2 E_{\mathrm{COF}}
$$

where $E_{2 C O F}$ and $E_{C O F}$ are both the total energies of optimized interlayers and single layer of COF, respectively. The large negative value means the strong interaction between two layers. The interlayer distance of COF was measured from the distance between the centroid of each layer.

Cell culture. Hep G2 cells were cultured in DMEM (high glucose) medium supplemented with $10 \%$ fetal bovine serum (FBS) with $100 \mathrm{U} / \mathrm{mL}$ penicillin and $0.1 \mathrm{mg} / \mathrm{mL}$ streptomycin at $37^{\circ} \mathrm{C}$ in a humidified $5 \% \mathrm{CO}_{2}$ incubator (HERAcell 150i). Subculture was performed every two days; for general cell culture, $25 \mathrm{~cm}^{2}$ of cell culture flask was used. Briefly, cells were digested using $0.25 \%$ trypsin $(1 \mathrm{~mL})$ containing $0.02 \%$ EDTA solution at $37^{\circ} \mathrm{C}$ for $3 \mathrm{~min}$, then complete cell culture medium $(2 \mathrm{~mL})$ was added and cells were gently piped off at bottom of culture flask. Cell suspensions were centrifuged at $100 \mathrm{~g}$ for $3 \mathrm{~min}$, the supernatant was discarded, and the complete culture medium $(3 \mathrm{~mL})$ was added and piped to obtain a single cell suspension. Cells suspension $(1 \mathrm{~mL})$ was transferred to a new $25-\mathrm{cm}^{2}$ cell culture flask, and the complete culture medium $(4 \mathrm{~mL})$ was added to re-suspend cells. After that, it was placed in $\mathrm{CO}_{2}$ cell incubator for further culture.

Cell viability test. Hep G2 cells were seeded in 96-well plates at the density of 5000 cells per well, wherein volumes of culture medium were all kept at $100 \mu \mathrm{L}$. After being incubated for $24 \mathrm{~h}$, culture medium was replaced with those medium containing the dispersion of $\mathrm{CON}(\mathrm{TpBD})$. The cell viability at $24 \mathrm{~h}$ and $48 \mathrm{~h}$ were determined by CCK8 assay, respectively.

Cellular uptake of CON-based samples. Hep G2 cells were seeded in 6-well culture dish at $2 \times 10^{5}$ cells per well for 24 -h incubation at $37^{\circ} \mathrm{C}$. Then BSA-FITC@CON was dispersed in PBS buffer to keep the concentration at $20 \mu \mathrm{g} / \mathrm{mL}$. Prior to the cultivation of samples with cells, pretreatment of the cells was carried out as follows: (1) the culture medium kept at $4^{\circ} \mathrm{C}$, instead of the regular $37^{\circ} \mathrm{C}$ condition; (2) $10 \mathrm{mM} \mathrm{NaN}_{3}$ in PBS buffer was added to treat the cells for $30 \mathrm{~min}$ at $37^{\circ} \mathrm{C}$, in order to reduce the intracellular ATP level; (3) $0.45 \mathrm{M}$ sucrose in PBS buffer was added to treat the cells for $30 \mathrm{~min}$ at $37^{\circ} \mathrm{C}$, in order to disrupt the formation of clathrin-coated vesicles. After that, the mixture in the presence of BSA-FITC@CON was incubated for 1.5 h. The cell culture medium was then discarded, and the residues were washed with PBS for three times to remove the remaining BSA-FITC@ $\mathrm{CON}$. Cells were digested and centrifuged, and then analyzed by the flow cytometry measurement data.

Characterization. Detailed morphological and structural characterizations were carried out by using high-resolution transmission electron microscope (HR TEM) (JEOL $2100 \mathrm{~F}$, Japan), operated at $200 \mathrm{kV}$ accelerating voltage at room temperature. The elemental mappings of $\mathrm{Fe}, \mathrm{N}$, and $\mathrm{O}$ atoms were collected using the same transmission electron microscope (operating at $200 \mathrm{kV}$ ) under the ADF STEM mode. FT IR spectra were recorded on Nicolet 6700 (Thermofisher, USA) Fourier transformation infrared spectrometer. Samples were dried and mixed with $\mathrm{KBr}$ to be compressed to a plate for measurement. ${ }^{1} \mathrm{H}$ NMR spectra were recorded on Varian Mercury plus $400 \mathrm{MHz}$ spectrophotometer at $298 \mathrm{~K}$. Solution-phase X-ray diffraction (PXRD) patterns were collected on an X-ray diffraction spectrometer (Bruker D8 Advance, Germany) with Cu Ko radiation at $\lambda=0.154 \mathrm{~nm}$ operating at $40 \mathrm{kV}$ and $40 \mathrm{~mA}$. Hydrodynamic diameters were conducted with a Nano ZS Zetasizer (model ZEN3600, Malvern Instruments) using a He-Ne laser at a wavelength of $632.8 \mathrm{~nm}$. Fluorescence spectra 
were obtained on a FLS920 spectrometer (Edinburgh Instruments). The morphology and height image of samples were obtained by scanning probe microscope (Bruker, Multimode 8). Flow cytometry measurement was carried out by flow cytometer (Beckman Coulter, A41). $\mathrm{N}_{2}$ adsorption-desorption isotherms were collected by a TriStar II 3020 volumetric adsorption analyzer (Micromeritics, USA) at $77 \mathrm{~K}$. The samples were degassed at $200^{\circ} \mathrm{C}$ for $24 \mathrm{~h}$ before measurement. Pore-size distribution was analyzed by non-local density functional theory (NLDFT) with $\mathrm{N}_{2}$-Carbon kernel based on a slit-pore model.

\section{References}

1. Feng, X., Ding, X. \& Jiang, D. Covalent organic frameworks. Chem. Soc. Rev. 41, 6010-6022 (2012).

2. Waller, P. J., Gándara, F. \& Yaghi, O. M. Chemistry of Covalent Organic Frameworks. Acc. Chem. Res. 48, 3053-3063 (2015).

3. Fang, Q. et al. 3D Porous crystalline polyimide covalent organic frameworks for drug delivery. J. Am. Chem. Soc. 137, 8325-8355 (2015).

4. Bai, L. et al. Nanoscale covalent organic frameworks as smart carriers for drug delivery. Chem. Commun. 52, 4128-4131 (2016).

5. Vyas, V. S. et al. Exploiting Noncovalent Interactions in an Imine-Based Covalent Organic Framework for Quercetin Delivery. Adv. Mater. 28, 8749-8754 (2016).

6. Mitra, S. et al. Targeted drug delivery in covalent organic Nanosheets (CONs) via sequential postsynthetic modification. J. Am. Chem. Soc. 139, 4513-4520 (2017).

7. Tan, J. et al. Manipulation of amorphous-to-crystalline transformation: Towards the construction of covalent organic framework hybrid microspheres with NIR photothermal conversion ability. Angew. Chem. Int. Ed. 55, 13979-13984 (2016).

8. Kandambeth, S. et al. Self-templated chemically stable hollow spherical covalent organic framework. Nat. Commun. 6, 6786 (2015).

9. Peng, Y. et al. Ultrathin two-dimensional covalent organic framework nanosheets: preparation and application in highly sensitive and selective DNA detection. J. Am. Chem. Soc. 139, 8698-8704 (2017).

10. Bisbey, R. P. \& Dichtel, W. R. Covalent organic frameworks as a platform for multidimensional polymerization. ACS Cent. Sci. 3 , 533-543 (2017).

11. Biswal, B. P. et al. Mechanochemical synthesis of chemically stable isoreticular covalent organic Frameworks. J. Am. Chem. Soc. 135, 5328 (2013).

12. Wang, S. et al. Exfoliation of covalent organic frameworks into few-layer redox-active nanosheets as cathode materials for lithiumion batteries. J. Am. Chem. Soc. 139, 4258-4261 (2017).

13. Berlanga, I. et al. Delamination of Layered Covalent Organic Frameworks. Small 7, 1207-1211 (2011).

14. Berlanga, I., Mas-Ballesté, R. \& Zamora, F. Tuning delamination of layered covalent organic frameworks through structural design. Chem. Commun. 48, 7976-7978 (2012).

15. Khayum, M. A. et al. Chemically Delaminated Free-Standing Ultrathin Covalent Organic Nanosheets. Angew. Chem. Int. Ed. 55, 15604-15608 (2016).

16. Mitra, S. et al. Self-exfoliated guanidinium-based ionic covalent organic nanosheets (iCONs). J. Am. Chem. Soc. 138, 2823-2828 (2016).

17. Dai, W. et al. Synthesis of a two-dimensional covalent organic monolayer through dynamic imine chemistry at the air/water interface. Angew. Chem. Int. Ed. 55, 21-217 (2016).

18. Chong, J. H., Sauer, M., Patrick, B. O. \& MacLachlan, M. J. Highly stable keto-enamine salicylideneanilines. Org. Lett. 5, 3823-3826 (2003).

19. Feldscher, B., Stammler, A., Bögge, H. \& Glaser, T. Synthesis and characterization of a trinuclear $\mathrm{Cu}_{3}{ }_{3} \mathrm{Complex}^{\mathrm{I}}$ bridged by an extended phloroglucinol-ligand: implications for a rational enhancement of ferromagnetic interactions. Dalton Trans. 39, 11675-11685 (2010).

20. Ding, X. et al. Synthesis of metallophthalocyanine covalent organic frameworks that exhibit high carrier mobility and photoconductivity. Angew. Chem. Int. Ed. 50, 1289-1293 (2011).

21. Feng, X. et al. High-rate charge-carrier transport in porphyrin covalent organic frameworks: switching from hole to electron to ambipolar conduction. Angew. Chem. Int. Ed. 51, 2618-2622 (2012).

22. Baldwin, L. A., Crowe, J. W., Pyles, D. A. \& McGrier, P. L. Metalation of a mesoporous three-dimensional covalent organic framework. J. Am. Chem. Soc. 138, 15134-15137 (2016).

23. Han, X. et al. Chiral covalent organic frameworks with high chemical stability for heterogeneous asymmetric catalysis. J. Am. Chem. Soc. 139, 8693-8697 (2017).

24. Li, L.-H. et al. Salen-based covalent organic framework. J. Am. Chem. Soc. 139, 6042-6045 (2017).

25. Bunck, D. N. \& Dichtel, W. R. Bulk synthesis of exfoliated two-dimensional polymers using hydrazone-linked covalent organic frameworks. J. Am. Chem. Soc. 135, 14952-14955 (2013).

26. Kandambeth, S. et al. Construction of crystalline $2 \mathrm{D}$ covalent organic frameworks with remarkable chemical (acid/base) stability via a combined reversible and irreversible route. J. Am. Chem. Soc. 134, 19524-19527 (2012).

27. Lee, D. S. et al. Selective protein transport through ultra-thin suspended reduced graphene oxide nanopores. Nanoscale 9 , 13457-13464 (2017).

28. Choudhary, P. et al. Fabrication of Non-toxic reduced Graphene Oxide Protein Nano-framework as Sustained Antimicrobial Coating for Biomedical Application. ACS Appl. Mater. Interfaces 9, 38255-38269 (2017).

29. $\mathrm{Hu}$, W. et al. Protein corona-mediated mitigation of cytotoxicity of graphene oxide. Acs Nano 5, 3693-700 (2011).

30. Jung, S. et al. Bio-functionalization of metal-organic frameworks by covalent protein conjugation. Chem. Commun. 47, 2904-2906 (2011).

31. Schmid, S. L. \& Carter, L. L. ATP is required for receptor-mediated endocytosis in intact cells. J. Cell Biol. 111, 2307 (1990).

32. Murkherjee, S., Ghosh, R. N. \& Maxfield, F. R. Endocytosis. Phys. Rev. 77, 759-803 (1997).

33. Heuser, J. E. \& Anderson, R. G. Hypertonic media inhibit receptor-mediated endocytosis by blocking clathrin-coated pit formation. J. Cell Biol. 108, 389 (1989).

34. Foster, J. S. et al. Gelation landscape engineering using a multi-reaction supramolecular hydrogelator system. J. Am. Chem. Soc. 137, 14236-14239 (2015).

35. Huang, N., Krishna, R. \& Jiang, D. Tailor-made pore surface engineering in covalent organic frameworks: systematic functionalization for performance screening. J. Am. Chem. Soc. 137, 7079-7082 (2015).

36. Sun, H. COMPASS: An ab initio force-field optimized for condensed-phase applications overview with details on alkane and benzene compounds. J. Phys. Chem. B 102, 7338-7364 (1998).

\section{Acknowledgements}

This work was financially supported by National Natural Science Foundation of China (No. 21474015 and 21774023) and Science and Technology Commission of Shanghai Municipality (No. 14ZR1402300). 


\section{Author Contributions}

W.K. carried out the experiments and analyzed the data. J.W. conducted the cellular experiments. S.N. performed the theoretical calculations. J.G. and W.K. wrote the manuscript. C.W. gave suggestions for analysis of experimental results. J.G. initiated, supervised and conceived the project. All authors read and approved the manuscript.

\section{Additional Information}

Supplementary information accompanies this paper at https://doi.org/10.1038/s41598-018-23744-1.

Competing Interests: The authors declare no competing interests.

Publisher's note: Springer Nature remains neutral with regard to jurisdictional claims in published maps and institutional affiliations.

(c) (i) Open Access This article is licensed under a Creative Commons Attribution 4.0 International License, which permits use, sharing, adaptation, distribution and reproduction in any medium or format, as long as you give appropriate credit to the original author(s) and the source, provide a link to the Creative Commons license, and indicate if changes were made. The images or other third party material in this article are included in the article's Creative Commons license, unless indicated otherwise in a credit line to the material. If material is not included in the article's Creative Commons license and your intended use is not permitted by statutory regulation or exceeds the permitted use, you will need to obtain permission directly from the copyright holder. To view a copy of this license, visit http://creativecommons.org/licenses/by/4.0/.

(C) The Author(s) 2018 